\title{
Minimum Weight Design of Composite Panel under Aeroelastic Constraint
}

\author{
Shinya Honda, ${ }^{\text {a* Gaku Yokoyama, }}{ }^{\mathrm{b}}$ \\ a'Department of Human Mechanical Systems \& Design, Faculty of Engineering, Hokkaido University. Email: honda @eng.hokudai.ac.jp \\ ${ }^{b}$ Department of Human Mechanical Systems \& Design, Faculty of Engineering, Hokkaido University.
}

\begin{abstract}
The present study minimizes the number of layers of wing shaped plates fabricated by laminated composite (CFRP) under aeroelastic constraint which keeps stable flutter speed. The finite element analysis (FEA) for the aeroelastic characteristics requires considerable calculation effort. Therefore, the response surface method (RSM) is utilized to reduce the computational time for optimization where an approximate expression uses bending rigidity of composite plates as input and flutter speed as output. Then, distributed genetic algorithm (DGA) is employed as an optimizer and minimizes numbers of layers of composite plates by designing fiber orientation angles. The optimum wing model shows large amount of weight reduction and enough flutter speed in less computational time than without RSM.
\end{abstract}

Keywords: Aeroelastic flutter; genenetic algorithm; laminated composite; optimum design

\section{Introduction}

In aerospace industry, weight saving of airframes is the most important issue due to increasing fuel costs, and use of composite materials, especially carbon fiber reinforced plastics (CFRP), are mainstream of aircraft development. CFRP are utilized for structures by stacking orthotropic layers because they have high specific strength and stiffness [1]. Design of its lay-up configurations enables aeroelastic tailoring, which controls stiffness of wings to meet aeroelastic requirements. Aeroelasticity is a field of study focusing on interactions among aerodynamic force, elastic force and inertia force for airframes [2]. Weisshaar [3] showed that composite materials are superior in aeroelastic tailoring. In aeroelastic problems, flutter [4] is critical in particular. Kameyama and Fukunaga [5] observed flutter properties of composite plates by using lamination parameters. Guo [6] optimized composite wings to satisfy weight and flutter requirements. These reports conducted aeroelastic tailoring for composite wings, however, there are few publications that aim at weight minimization of composite wings and search practical lay-up configurations in consideration of lay-up constraint.

The present paper proposes the practical optimization method for lightweight aircrafts. Distributed Genetic Algorithm (DGA) [7] is employed as an optimizer. In the optimization, fiber orientation angles of laminated composites are assigned to design variables, and the critical value of flutter speed is imposed as the constraint.

The optimization problem requires a lot of calculation effort for aerodynamic analyses of the wing shaped models by the finite element analysis (FEA). Therefore, Response Surface Method (RSM) is involved in the optimization. The RSM is available to various optimization problems in order to reduce computational cost. Todoroki and Ishikawa [8] studied the optimization of laminate stacking sequence for maximizing buckling load with Genetic Algorithm (GA) and the RSM. The optimum wing model obtained by the optimization in less computational time showed the effective weight reduction and enough resistance to the flutter. The present study employs MD. Nastran for aerodynamic analysis and MATLAB for other optimization process.

\footnotetext{
${ }^{*}$ Corresponding author. Tel.: +81-11-706-6415

Kita-13, Nishi-8, Kita-ku

Sapporo, Hokkaido, Japan, 060-8628
} 

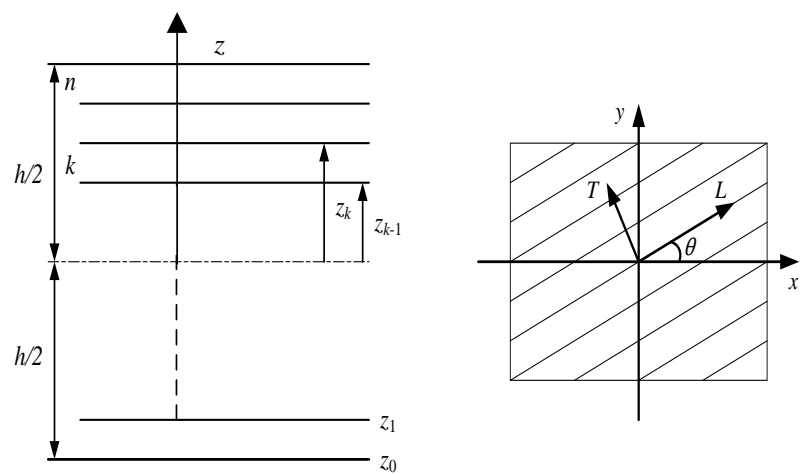

Figure 1. The coordinate systems of laminated plates. and minor Poisson rations, $z_{k}$ is the distance from the plate middle surface to the upper surface of the $k$ th layer, and $\theta$ is the fiber orientation angle in the $k$ th layer.

\subsection{Finite element analysis}

The finite element model with four-node elements as shown in Fig. 2 is used for structural and aerodynamic finite element analyses (FEA) by MD. Nastran. In structural analysis, the lowest four vibration mode shapes and natural frequencies are calculated. In aerodynamic analysis, critical air speed leading to flutter $V_{F}$ is evaluated with the $\Delta V_{F}=1$ $\mathrm{m} / \mathrm{s}$. Dimensions of this model and material constants for CFRP are defined as

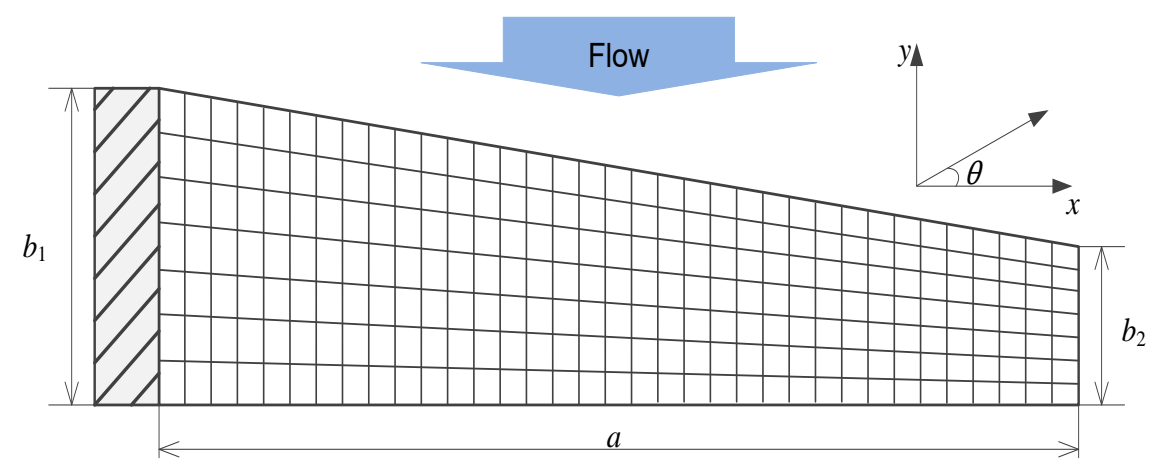

Figure 2. The finite element mesh model.

\section{Analysis method}

\subsection{Bending stiffness of laminated plates}

A symmetrically $N$-laminated plate is considered as shown in Fig. 1. The bending stiffness $D_{i j}$ of this plate is given by

$$
\begin{aligned}
& D_{i j}=\frac{1}{3} \sum_{k=1}^{N}\left(\bar{Q}_{i j}\right)_{k}\left(z_{k}^{3}-z_{k-1}^{3}\right), \quad(i, j=1,2,6) \\
& \bar{Q}_{11}=Q_{11} l^{4}+2\left(Q_{12}+2 Q_{66}\right) l^{2} m^{2}+Q_{22} m^{4} \\
& \bar{Q}_{12}=Q_{12}\left(l^{4}+m^{4}\right)+\left(Q_{11}+Q_{22}-4 Q_{66}\right) l^{2} m^{2} \\
& \bar{Q}_{22}=Q_{11} m^{4}+2\left(Q_{12}+2 Q_{66}\right) l^{2} m^{2}+Q_{22} l^{4} \\
& \bar{Q}_{16}=\left(Q_{11}-Q_{12}-2 Q_{66}\right) l^{3} m-\left(Q_{22}-Q_{12}-2 Q_{66}\right) l m^{3} \\
& \bar{Q}_{26}=\left(Q_{11}-Q_{12}-2 Q_{66}\right) l m^{3}-\left(Q_{22}-Q_{12}-2 Q_{66}\right) l^{3} m \\
& \bar{Q}_{66}=\left(Q_{11}+Q_{22}-2 Q_{12}-2 Q_{66}\right) l^{2} m^{2}+Q_{66}\left(l^{4}+m^{4}\right) \\
& Q_{11}=\frac{E_{L}}{1-v_{L T} v_{T L}}, \quad Q_{22}=\frac{E_{T}}{1-v_{L T} v_{T L}}, \\
& Q_{12}=\frac{v_{T L} E_{L}}{1-v_{L T} v_{T L}}=\frac{v_{L T} E_{T}}{1-v_{L T} v_{T L}}, \\
& Q_{66}=G_{L T}, \quad l=\cos \theta, \quad m=\sin \theta
\end{aligned}
$$

where $E_{L}$ and $E_{T}$ are the moduli of elasticity in the $L$ and $T$ directions, $G_{L T}$ is the shear modulus, $v_{L T}$ and $v_{T L}$ are the major

$$
\begin{array}{ll}
a=1000 \mathrm{~mm}, & b_{1}=300 \mathrm{~mm}, \\
b_{2}=150 \mathrm{~mm}, & t=0.19 \mathrm{~mm} \\
E_{L}=152 \mathrm{GPa}, & E_{T}=8.00 \mathrm{GPa}, \\
G_{L T}=4.00 \mathrm{GPa} & \\
v_{L T}=0.340, & \rho=1600 \mathrm{~kg} / \mathrm{m}^{3}
\end{array}
$$

where the thickness of one ply is denoted by $t$.

In order to compare with optimized models, the benchmark model with $\left[45^{\circ} / 0^{\circ} /-45^{\circ} / 90^{\circ}\right]_{4 s}$ lay-up is introduced here. As a result of aerodynamic analysis, $V_{F}$ of the benchmark model is obtained as

$$
V_{\text {Frequired }}=165 \mathrm{~m} / \mathrm{s}
$$

\section{Response surface method}

RSM gives an approximate equation between responses $y$ and predictor variables $x$. The present study employs the response surface equation written as

$$
\begin{aligned}
& y=\beta_{0}+\beta_{1} x_{1}+\beta_{2} x_{2}+\beta_{3} x_{3}+\beta_{4} x_{4} \\
& +\beta_{5} x_{5}+\beta_{6} x_{6}+\beta_{7} x_{7}+\beta_{8} x_{8}+\beta_{9} x_{9} \\
& +\beta_{10} x_{10}+\beta_{11} x_{11}+\beta_{12} x_{12}+\beta_{13} x_{13} \\
& +\beta_{14} x_{14}+\beta_{15} x_{15}+\beta_{16} x_{16}+\beta_{17} x_{17} \\
& +\beta_{18} x_{18}+\beta_{19} x_{19}+\beta_{20} x_{20}
\end{aligned}
$$


with

$x_{1}=D_{11}, x_{2}=D_{16}, x_{3}=D_{22}, x_{4}=D_{26}$,

$x_{5}=D_{66}, x_{6}=D_{11}{ }^{2}, x_{7}=D_{16}{ }^{2}, x_{8}=D_{22}{ }^{2}$,

$x_{9}=D_{26}{ }^{2}, x_{10}=D_{66}{ }^{2}$,

$x_{11}=D_{11} \times D_{16}, x_{12}=D_{11} \times D_{22}$,

$x_{13}=D_{11} \times D_{26}, \quad x_{14}=D_{11} \times D_{66}$,

$x_{15}=D_{16} \times D_{22}, \quad x_{16}=D_{16} \times D_{26}$,

$x_{17}=D_{16} \times D_{66}, \quad x_{18}=D_{22} \times D_{26}$,

$x_{19}=D_{22} \times D_{66}, \quad x_{20}=D_{26} \times D_{66}$

Table 1. Values and F-statistics of the coefficients.

\begin{tabular}{lll}
\hline & Values & F-statistics \\
\hline$b_{0}$ & 154.0 & 1996270 \\
$b_{1}$ & 20.01 & 7330 \\
$b_{2}$ & -40.41 & 7680 \\
$b_{3}$ & 4.933 & 567.2 \\
$b_{4}$ & 22.92 & 2869 \\
$b_{5}$ & 49.51 & 21543 \\
$b_{6}$ & 5.388 & 184.0 \\
$b_{7}$ & -168.8 & 26486 \\
$b_{8}$ & 3.867 & 142.9 \\
$b_{9}$ & -72.15 & 6029 \\
$b_{10}$ & -8.355 & 155.7 \\
$b_{11}$ & -9.854 & 85.91 \\
$b_{12}$ & 4.720 & 96.53 \\
$b_{13}$ & 13.07 & 221.0 \\
$b_{14}$ & 4.470 & 37.65 \\
$b_{15}$ & -13.58 & 225.2 \\
$b_{16}$ & 112.6 & 7619 \\
$b_{17}$ & 9.373 & 45.33 \\
$b_{18}$ & -2.947 & 9.587 \\
$b_{19}$ & - & 0.02932 \\
$b_{20}$ & -5.311 & 16.86 \\
\hline & &
\end{tabular}

where $y, x$ and $\beta$ represent flutter speed, bending stiffness and unknown coefficients respectively. Here, $D_{12}$ is omitted due to linear relationship between $D_{12}$ and $D_{66}$. The linear multiple regression model is expressed as

$$
\begin{array}{ll}
\mathbf{y}=\mathbf{X} \boldsymbol{\beta} \boldsymbol{\varepsilon} & \left\{\begin{array}{c}
y_{1} \\
y_{2} \\
\vdots \\
y_{n}
\end{array}\right\}, \\
\boldsymbol{\beta}=\left\{\begin{array}{c}
\beta_{0} \\
\beta_{1} \\
\vdots \\
\beta_{k}
\end{array}\right\}, & \mathbf{X}=\left[\begin{array}{ccccc}
1 & x_{11} & x_{12} & \cdots & x_{1 k} \\
1 & x_{21} & x_{22} & \cdots & x_{2 k} \\
\vdots & \vdots & \vdots & \ddots & \vdots \\
1 & x_{n 1} & x_{n 2} & \cdots & x_{n k}
\end{array}\right] \\
& \quad \boldsymbol{\varepsilon}=\left\{\begin{array}{c}
\varepsilon_{1} \\
\varepsilon_{2} \\
\vdots \\
\varepsilon_{n}
\end{array}\right\}
\end{array}
$$

where $n$ is the number of sampling point, $k$ is the number of variables, and $\varepsilon$ is an error vector. The unbiased estimator $b$ of $\beta$ is obtained by the least square method as follows [8].

$$
\begin{aligned}
& \mathbf{b}=\left(\mathbf{X}^{\mathrm{T}} \mathbf{X}\right)^{-1} \mathbf{X}^{\mathrm{T}} \mathbf{y} \\
& \mathbf{b}=\left\{\begin{array}{c}
b_{0} \\
b_{1} \\
\vdots \\
b_{k}
\end{array}\right\}
\end{aligned}
$$

The RSM equation is created along the following three steps.

1. The database of $V_{F}$ derived random bending stiffness of the wing model shown in Fig. 2 is created by aerodynamic analysis. In order to create this database, 4500 times of FEA trials are conducted. Then, the data quantity is reduced to 3923 due to erasing of overlapped data, and the values of bending stiffness are normalized based on the maximum and minimum value.

2. The unbiased estimator $\mathbf{b}$ is given by Eq. (6) for the database, where $k=20$ and $n=3923$.

3. F-test is performed to verify the validity of $\mathbf{b}$ with significance level 0.01 as shown in Table 1. Finally, the response surface equation is written as follows.

$$
\begin{aligned}
& y=154.0+20.01 x_{1}-40.41 x_{2}+4.933 x_{3} \\
& +22.92 x_{4}+49.51 x_{5}+5.388 x_{6}-168.8 x_{7} \\
& +3.867 x_{8}-72.15 x_{9}-8.355 x_{10}-9.854 x_{11} \\
& +4.720 x_{12}+13.07 x_{13}+4.470 x_{14}-13.58 x_{15} \\
& +112.6 x_{16}+9.373 x_{17}-2.947 x_{18}-5.311 x_{20}
\end{aligned}
$$

The adjusted determination coefficient is 0.8915 and indicates excellent accuracy of this equation.

\section{Optimization method}

Distributed Genetic Algorithm (DGA) [7] is adopted to solve the stacking sequence optimization problem as follows.

$$
\begin{array}{ll}
\text { [Objective function] } & \text { Minimize } n(1 \leq n \leq 32) \\
\text { [Subject to] } & V_{F} \geq V_{F \text { required }} \\
& \text { Symmetric lamination } \\
& \text { Less than } 3 \text { consecutive } \\
& \text { lamination of the same } \theta \\
& \theta_{i}(i=1,2, \ldots, n / 2) \\
\text { [Design variable] } & -85 \leq \theta \leq 90\left(\Delta \theta=5^{\circ}\right)
\end{array}
$$

In this DGA, a large population is distributed to some islands labeled by each number of lamination. Thus, weight reduction is performed by selection of labeled islands as the number of lamination. At the same time, lay-up configurations in each island are optimized. Fitness $H$ is evaluated independently in each islands as follows. 


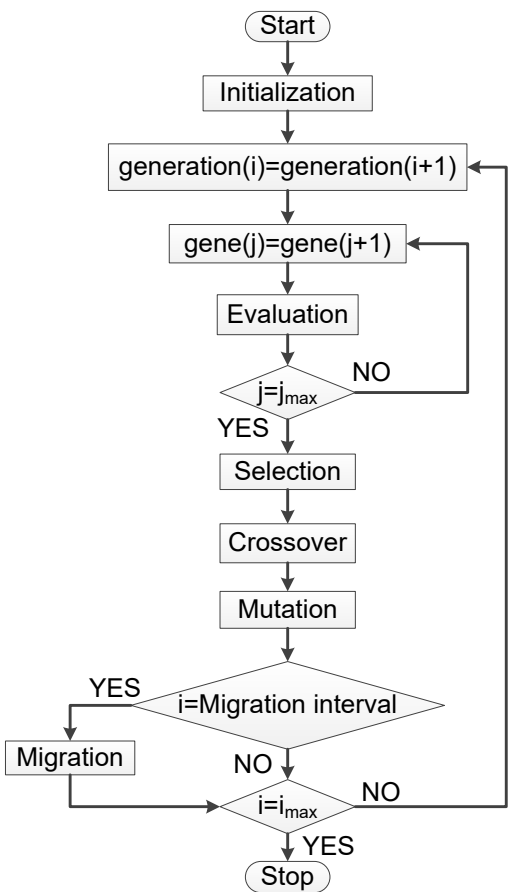

Figure 3. Flowchart of DGA

$$
H=V_{F}
$$

Moreover, DGA has the unique operation named as migration. This operation exchanges some individuals among some islands, and it contributes to keep diversity of populations. As DGA parameters, the number of generation is 100 , total population is 150 , crossover rate is 0.9 , mutation rate is 0.1 , the number of islands in one generation is two and migration interval is 10 . This optimization adopts elitist strategy, roulette wheel selection and 2-point crossover. Fig. 3 illustrates flowchart of DGA. Table 2 represents decoding in DGA.

The optimization described above is performed in two cases Opt. A and Opt. B. In Opt. A, $V_{F}$ is calculated by FEA in each time. On the other hand, estimated value $y$ of Eq. (7) is substituted for $V_{F}$ to reduce computational load in Opt. B.

\section{Results and discussions}

Table 3 shows the comparison of the benchmark model and two optimum models. The optimum solutions in Opt. A and $\mathrm{B}$ present $12.5 \%$ reduced weight from the benchmark model and keep enough $V_{F}$. In Opt. B, $y=179 \mathrm{~m} / \mathrm{s}$ is derived as the optimum solution with estimation equation. The calculated value by FEA for the obtained solution is $176 \mathrm{~m} / \mathrm{s}$, and the error is only $1.70 \%$ in spite of $70 \%$ reduction of FEA trials. Since the lay-up configurations of the optimum models contain the angles strong to torsion such as $45^{\circ},-45^{\circ}$, $40^{\circ}$ and $-40^{\circ}$, it is revealed that the optimum solutions have high $D_{66}$ values, resulting in better resistance to flutter. Moreover, the values of $D_{16}$ decrease compared to the benchmark model. These results indicate the importance of
Table 2. Decoding

\begin{tabular}{|c|c|c|}
\hline Senary & Decimal & $\begin{array}{c}\text { Phenotype } \\
\text { (Fiber orientation angle) }\end{array}$ \\
\hline 00 & 0 & 0 \\
\hline 01 & 1 & 5 \\
\hline 11 & 7 & 10 \\
\hline 10 & 6 & 15 \\
\hline 12 & 8 & 20 \\
\hline 02 & 2 & 25 \\
\hline 22 & 14 & 30 \\
\hline 21 & 13 & 35 \\
\hline 20 & 12 & 40 \\
\hline 23 & 15 & 45 \\
\hline 13 & 9 & 50 \\
\hline 03 & 3 & 55 \\
\hline 33 & 21 & 60 \\
\hline 32 & 20 & 65 \\
\hline 31 & 19 & 70 \\
\hline 30 & 18 & 75 \\
\hline 34 & 22 & 80 \\
\hline 24 & 16 & 85 \\
\hline 14 & 10 & 90 \\
\hline 04 & 4 & -85 \\
\hline 44 & 28 & -80 \\
\hline 43 & 27 & -75 \\
\hline 42 & 26 & -70 \\
\hline 41 & 25 & -65 \\
\hline 40 & 24 & -60 \\
\hline 45 & 29 & -55 \\
\hline 35 & 23 & -50 \\
\hline 25 & 17 & -45 \\
\hline 15 & 11 & -40 \\
\hline 05 & 5 & -35 \\
\hline 55 & 35 & -30 \\
\hline 54 & 34 & -25 \\
\hline 53 & 33 & -20 \\
\hline 52 & 32 & -15 \\
\hline 51 & 31 & -10 \\
\hline 50 & 30 & -5 \\
\hline
\end{tabular}

$D_{66}$ and unimportance of $D_{16}$ as observed from the Fstatistics of $b_{5}$ and $b_{7}$ in Table 1.

Figures 4-6 show natural frequencies and vibration mode shapes for Bench mark model and both solutions of Opt. A and B. It is known from Figs. 4-6 that the natural frequencies for the bending modes decrease and torsional modes increase compared with the benchmark model since the layup configurations are strengthened against torsion.

\section{Conclusions}

The present study proposed the optimization method to reduce weight of wing shaped composite plates by using DGA and RSM. This method yielded the excellent optimum solution precisely in less computational time compared to the case without RSM. The obtained results revealed that assigning bending stiffness to predictor variables is effective to derive $V_{F}$ as responses for RSM. In addition, the tested coefficients of the response surface equation showed the significant factors to keep enough $V_{F}$. 
Table 3. Comparison between three models

\begin{tabular}{|c|c|c|c|}
\hline & $\begin{array}{c}\text { The } \\
\text { benchmark } \\
\text { model }\end{array}$ & Opt. A & Opt. B \\
\hline Lay-up & $\begin{array}{l}{[45 / 0 /-} \\
45 / 90]_{4 \mathrm{~s}}\end{array}$ & $\begin{array}{c}{[-40 / 45 / 45 /-} \\
45 /-30 / 40 /-40 / \\
35 /-65 /-50 /-5 /- \\
40 / 0 /-65]_{\mathrm{s}}\end{array}$ & $\begin{array}{c}{[-40 / 40 / 45 /-40 /-} \\
40 / 45 /-40 / \\
30 /- \\
40 / 45 / 10 / 45 / 65 / 25]_{\mathrm{s}}\end{array}$ \\
\hline$V_{F}[\mathrm{~m} / \mathrm{s}]$ & 165 & 175 & 176 \\
\hline$D_{11}[\mathrm{~N} \cdot \mathrm{m}]$ & 1197 & 708.6 & 731.5 \\
\hline$D_{12}[\mathrm{~N} \cdot \mathrm{m}]$ & 385.5 & 441.0 & 452.1 \\
\hline$D_{16}[\mathrm{~N} \cdot \mathrm{m}]$ & 19.87 & -56.13 & -32.49 \\
\hline$D_{22}[\mathrm{~N} \cdot \mathrm{m}]$ & 1149 & 498.0 & 452.9 \\
\hline$D_{26}[\mathrm{~N} \cdot \mathrm{m}]$ & 19.87 & -19.22 & -1.610 \\
\hline$D_{66}[\mathrm{~N} \cdot \mathrm{m}]$ & 409.2 & 456.9 & 468.0 \\
\hline $\begin{array}{l}\text { The number } \\
\text { of layers }\end{array}$ & 32 & 28 & 28 \\
\hline Weight $[\mathrm{kg}]$ & 2.189 & 1.915 & 1.915 \\
\hline $\begin{array}{l}\text { Reduced } \\
\text { weight [\%] }\end{array}$ & $\begin{array}{l}\text { Reference } \\
\text { value }\end{array}$ & 12.5 & 12.5 \\
\hline $\begin{array}{l}\text { aerodynamic } \\
\text { FEA trials }\end{array}$ & - & 15000 & 4500 \\
\hline $\begin{array}{c}\text { Reduced } \\
\text { FEA trials } \\
{[\%]}\end{array}$ & - & $\begin{array}{l}\text { Reference } \\
\text { value }\end{array}$ & 70 \\
\hline
\end{tabular}

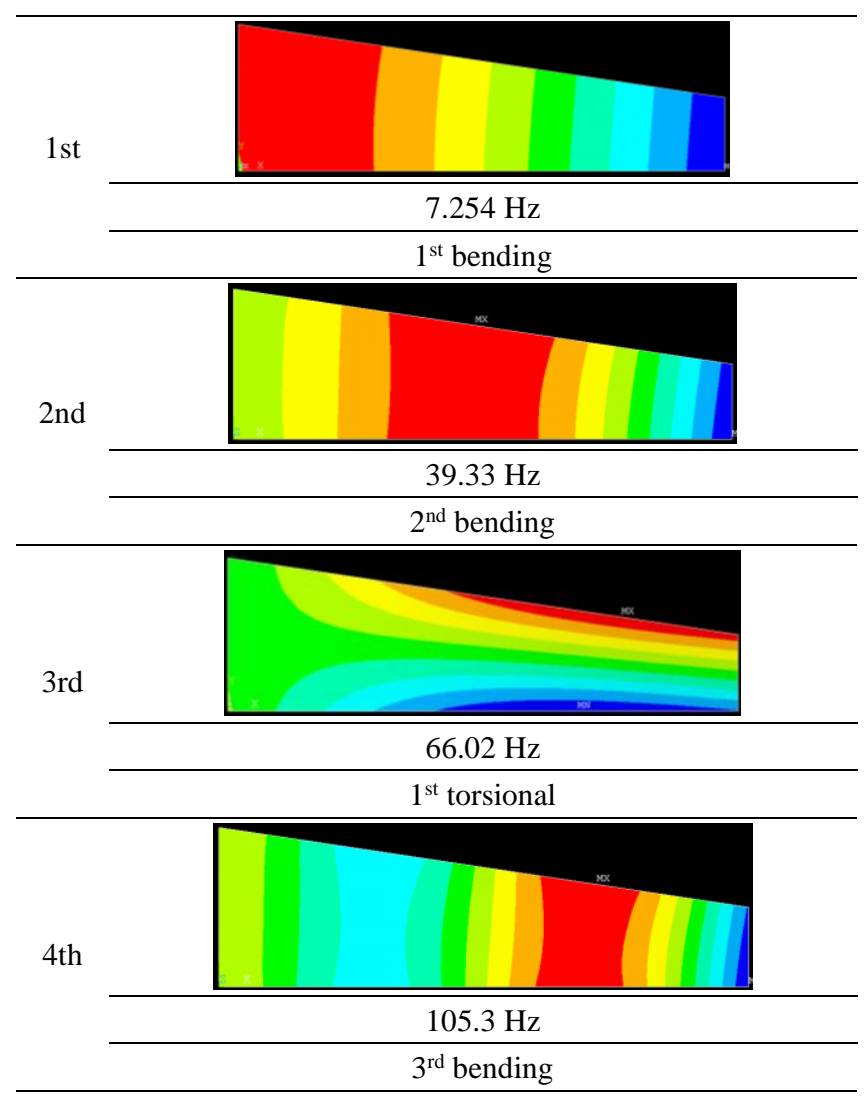

Figure 4. Vibration mode shapes and natural frequencies for benchmark model

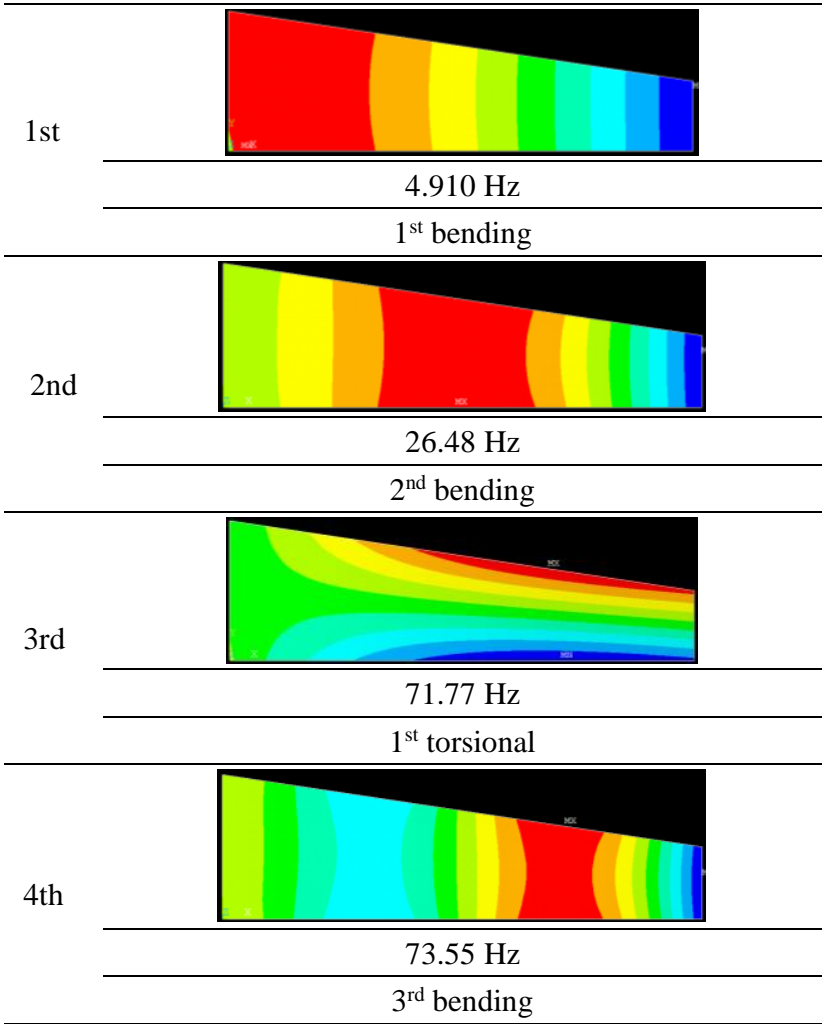

Figure 5. Vibration mode shapes and natural frequencies for Opt A

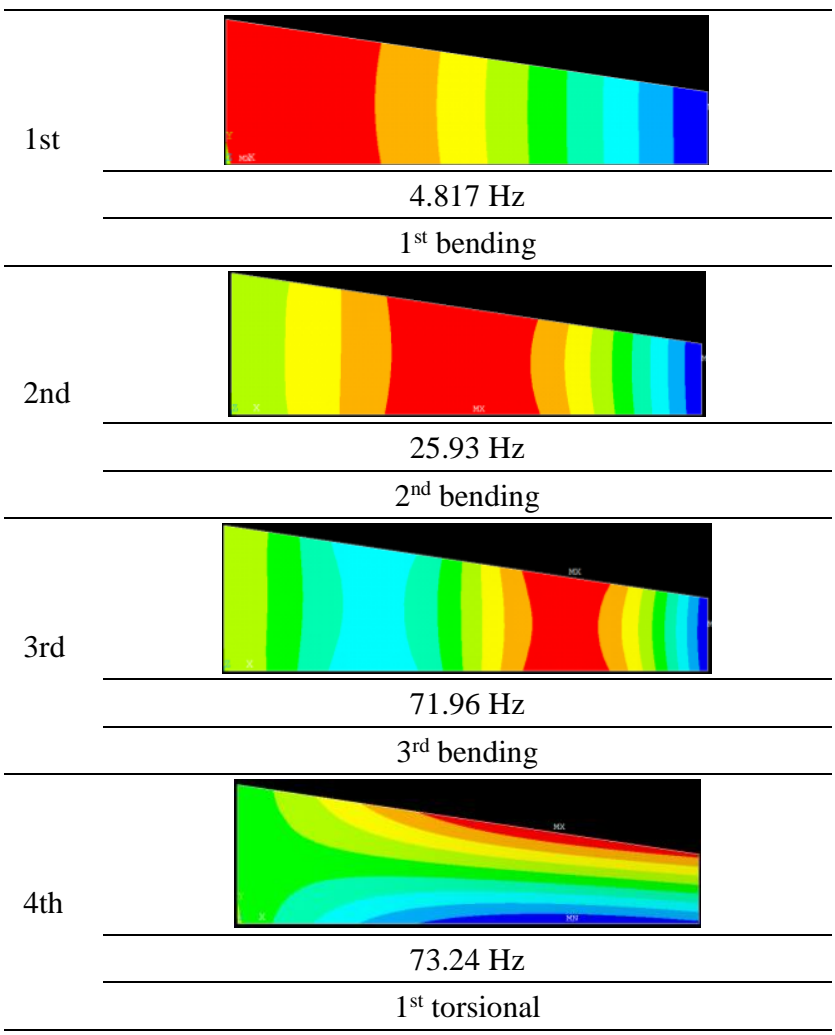

Figure 6. Vibration mode shapes and natural frequencies for Opt B 


\section{References}

[1]. Fukuda, H. and Ben, G. 1989. "Introduction of dynamics of composite materials," Kokonshoin (in Japanese).

[2]. Washizu, K. 1957. "Aeroelastics," Kyoritsu (in Japanese).

[3]. Weisshaar, T. A.. 1994. "Active Tailoring with Advanced Materials," Journal of the Japan Society for Aeronautical and Space Sciences, 42(487):455-461.

[4]. Bisplinghoff, R. L., Ashley, H., and Halfman. R. L., 1996. "Aeroelasticity," Dover Publications.

[5]. Kameyama, M. and Fukunaga, H. 2007. "Optimum Design of Composite Plate Wings for Aeroelastic Characteristics Using Lamination Parameters," Computers \& Structures, 85:213-224.
[6]. Guo, S.. 2007. "Aeroelastic Optimization of an Aerobatic Aircraft Wing Structure," Aerospace Science and Technology, 11:396-404.

[7]. Hiroyasu, T., Miki, M. and Negami, M. 1999. "Distributed Genetic Algorithms with Randomized Migration Rate," in IEEE Proceedings of Systems, Man and Cybernetics Conference SMC'99, 1:689-694.

[8]. Todoroki, A. and Ishikawa, T. 2004. "Design of Experiments for Stacking Sequence Optimizations with Genetic Algorithm Using Response Surface Approximation," Composite Structures, 64:349-357. 